\title{
Difference boundary value problem hierarchies and the forward Crum transformation
}

Sonja Currie* and Anne D Love

\section{"Correspondence:}

Sonja.Currie@wits.ac.za

School of Mathematics, University

of the Witwatersrand,

Johannesburg, Private Bag 3, Wits,

2050, South Africa

\begin{abstract}
This paper uses the forward transformation defined in Currie and Love (Adv. Differ. Equ. 2010:947058, 2010) to develop a hierarchy for difference boundary value problems with eigenparameter-dependent boundary conditions. In particular, we show how various sets of these boundary conditions transform under this forward transformation. The resulting hierarchy is then illustrated in a tabular form where the number of eigenvalues for each of the problems is also given. In addition, we point out certain analogies to the work done in Currie and Love (Bound. Value Probl. 2011:743135, 2011) where the reverse Crum-type transformation was used to establish a hierarchy of difference boundary value problems.
\end{abstract}

MSC: 39A05; 34K10; 65Q10; 65Q30

Keywords: difference equations; hierarchies; boundary value problems; transformations; Nevanlinna; eigenvalues

\section{Introduction}

One of the main reasons for studying the Darboux-Crum transformation on a discrete second-order difference equation is its relevance to quantum mechanics in the computation of discrete energy levels and corresponding eigenfunctions. This follows from the fact that a second-order difference equation can be factorised as a product of two Crum-type transformations, see [1].

This paper develops an alternative hierarchy to that given in [2] for difference boundary problems by using the forward Crum-type transformation that was given initially in [1]. Our interest in this alternate hierarchy is its value in the study of the associated inverse problem which is our current project. Although the results appear similar to those in [2-4], it should be noted that they do not follow directly from these papers and as such, it is necessary that these new results be proved as shown in Sections 3.1 and 3.2.

We consider a weighted second-order difference equation of the form

$$
c(n) y(n+1)-b(n) y(n)+c(n-1) y(n-1)=-c(n) \lambda y(n)
$$

where $c(n)>0$ represents a weight function and $b(n)$ a potential function. General eigenparameter-dependent boundary conditions of the form

O2013 Currie and Love; licensee Springer. This is an Open Access article distributed under the terms of the Creative Commons Attribution License (http://creativecommons.org/licenses/by/2.0), which permits unrestricted use, distribution, and reproduction in any medium, provided the original work is properly cited. 


$$
\begin{aligned}
& y(-1)=\left[g \lambda+h-\sum_{k=1}^{p} \frac{u_{k}}{\lambda-e_{k}}\right] y(0), \quad g \geq 0, u_{k}>0, \\
& y(m-1)=\left[a \lambda+b-\sum_{k=1}^{s} \frac{c_{k}}{\lambda-d_{k}}\right] y(m), \quad a \leq 0, c_{k}<0
\end{aligned}
$$

are imposed at the initial and terminal endpoints.

We prove how (1.2) transforms under transformation (2.1). It is necessary to consider the cases of $g=0$ and $g>0$ separately in order to establish our results (see Theorem 3.5). The majority of the work carried out in this paper pertains to the transformation of (1.3) under (2.1) (see Theorems 3.2, 3.3 and 3.4). Here we consider the cases $a=0$ and $a<0$ separately because when $a=0$ it is necessary to then further consider independently the cases of $b=0$ and $b \neq 0$. It should be noted that when $a<0$ the sign of $b$ is immaterial. Interestingly, in Theorem 3.4, the case when both $a=0$ and $b=0$ is unique in that the interval of the transformed boundary value problem shrinks by one unit. Furthermore, a comparison of the original and transformed boundary value problems and their corresponding eigenvalues is given in Tables 1 and 2 in Section 4. In addition, the analogies between the hierarchy presented in this paper and that found in [2-4] are mentioned.

In mathematics, the difference equation is often used as a means to study its continuous counterpart, the differential equation. However, difference equations are interesting and useful in their own right. Difference equations or recurrence relations describe a situation where there is a discrete sequence of entities, each of which either gives an input to its successor, or perhaps interacts with both its neighbors.

Difference equations have applications in the theory of orthogonal polynomials and continued fractions, electrical circuit analysis, signals and systems analysis, computer visualization, mathematical biology, dynamical systems and statistics etc. In particular, secondorder difference equations or three-term recurrence relations of the form (1.1) are used to model a variety of problems, see [5], such as the famous 'gamblers ruin' model in probability theory, the national income model in economics and the model for the propagation of annual plants in biology. Moreover, in dynamical systems, three-term recurrence relations are used to model population dynamics; for example, the Fibonacci numbers, which are defined by the relation

$$
y(n)=y(n-1)+y(n-2)
$$

with $y(0)=0$ and $y(1)=1$, were once used as a model for the growth of rabbit populations.

For an application of (1.1) with variable coefficients together with eigenparameterdependent boundary conditions, we turn our attention to quantum physics. Here $c(n)$ being a variable indicates that the medium or space considered is non-uniform. The coefficient $b(n)$, which may or may not vary, is the discrete analogue of the potential in the continuous case. The significance of the eigenparameter-dependent boundary conditions is that the boundary of the space responds to the energy of the system, i.e. is not fixed, where the eigenparameter $\lambda$ represents the energy level (up to a scaling factor). In particular, in the one-dimensional discrete model of an electron in an atom, $\lambda$ gives the energy state of the electron.

It should be noted that Harmsen and $\mathrm{Li}$, in [6], study discrete Sturm-Liouville problems where the eigenparameter appears linearly in the boundary conditions. These results are 
then extended by the authors in [7] to difference boundary value problems where the spectral parameter appears quadratically in the boundary conditions. The operator is extended to a self-adjoint operator and an expansion theorem is proved.

Difference equations are also being studied using Lie group theory. This usually involves a symmetry-based approach to solving a given ordinary difference equation by considering the structure of the solution set. A symmetry is a continuous group which leaves the system of equations invariant. For example, if a one-dimensional difference equation admits a symmetry, then it becomes a linear equation and an analytic solution may be obtained as described in [8] and [9]. Hydon in [10] develops a method for finding one-parameter Lie groups of symmetries to achieve successive reductions of order. The difference equation can be completely solved provided there are a sufficient number of symmetries. Levi and Winternitz in [11] show how the apparent mismatch between discrete equations and continuous symmetries can be resolved. Their approach is either to use generalised symmetries on the solutions of the difference equations which leave the lattice invariant or, alternatively, restrict to point symmetries which allow them to transform the lattice.

Section 2 provides the preliminary results from [1-4]. Section 3 pertains to the transformation of boundary conditions (1.2) and (1.3) under mapping (2.1) as discussed above, while Section 4 provides the reader with a tabularized comparison of various sets of boundary conditions and eigenvalues for the original boundary value problem with those of the transformed boundary value problem.

\section{Preliminaries}

This section provides a recap of the necessary results from [1-4].

Consider equation (1.1) for $n=0, \ldots, m-1$. Note that the values of $y(-1)$ and $y(m)$ are given by the boundary conditions.

The mapping $y \mapsto v$ is defined by

$$
v(n):=y(n+1)-y(n) \frac{z(n+1)}{z(n)}, \quad n=-1,0, \ldots, m-1,
$$

where, throughout this paper, $z(n)$ is a solution to (1.1) for $\lambda=0$ such that $z(n)>0$ for all $n=-1, \ldots, m$.

Note Without loss of generality:

(i) If $z(n)$ does satisfy both given boundary conditions (1.2) and (1.3), then the spectral parameter must be shifted so that the original boundary value problem has least eigenvalue precisely 0 .

(ii) If $z(n)$ does not obey one or both given boundary conditions (1.2) and (1.3), then the spectral parameter of the original given boundary value problem must first be shifted so as to ensure that all the eigenvalues are nonnegative.

(iii) Once the required shifts above have been made, $z(n)$ is taken to be a solution to the shifted equation, which we may assume is given again by (1.1) for $\lambda=0$.

From [1] we have the following theorem.

Theorem 2.1 Under mapping (2.1), equation (1.1) transforms to

$$
c_{v}(n) v(n+1)-b_{\nu}(n) v(n)+c_{\nu}(n-1) v(n-1)=-\lambda c_{\nu}(n) v(n),
$$


for $n=0, \ldots, m-2$, and where for $n=-1,0, \ldots, m-1$,

$$
\begin{aligned}
& c_{v}(n)=\frac{c(n) z(n)}{z(n+1)}>0, \\
& b_{v}(n)=\left[\frac{c(n) z(n)}{c(n+1) z(n+1)}+\frac{z(n+1)}{z(n)}\right] \frac{c(n) z(n)}{z(n+1)} .
\end{aligned}
$$

As in our previous research [2-4], Nevanlinna functions play an important role. For the reader's convenience, the particular properties of Nevanlinna functions that are employed are listed below.

(A) If $N(\lambda), B(\lambda)$ are positive Nevanlinna functions, then

$$
\frac{1}{N(\lambda)}=-B(\lambda)
$$

In particular, it follows from (A) that the results below hold:

(B) If

$$
N(\lambda)=b-\sum_{j=1}^{s} \frac{c_{j}}{\lambda-d_{j}}, \quad c_{j}>0, b \neq 0,
$$

then

$$
\frac{1}{N(\lambda)}=\beta-\sum_{j=1}^{s} \frac{\sigma_{j}}{\lambda-\delta_{j}}, \quad \sigma_{j}<0, \beta \neq 0 .
$$

(C) If

$$
N(\lambda)=a \lambda+b-\sum_{j=1}^{s} \frac{c_{j}}{\lambda-d_{j}}, \quad a, c_{j}>0
$$

then

$$
\frac{1}{N(\lambda)}=-\sum_{j=1}^{s+1} \frac{\sigma_{j}}{\lambda-\delta_{j}}, \quad \sigma_{j}<0 .
$$

The graph given in Figure 1 depicts the positive Nevanlinna function

$$
y(\lambda)=A \lambda+B-\sum_{k=1}^{s} \frac{\varrho_{k}}{\lambda-x_{k}}, \quad \varrho_{k}>0
$$

together with the horizontal line $y(\lambda)=K$, where the intersections are labeled by $\tau_{i}$ 's. For illustrative purposes, we have taken $s=3$. This graph will be referred to throughout Section 3.

The notation used will follow that in [2], namely $N_{s, j}^{\diamond}(\lambda)$ will denote a Nevanlinna function where:

$s$ is the number of terms in the sum; 


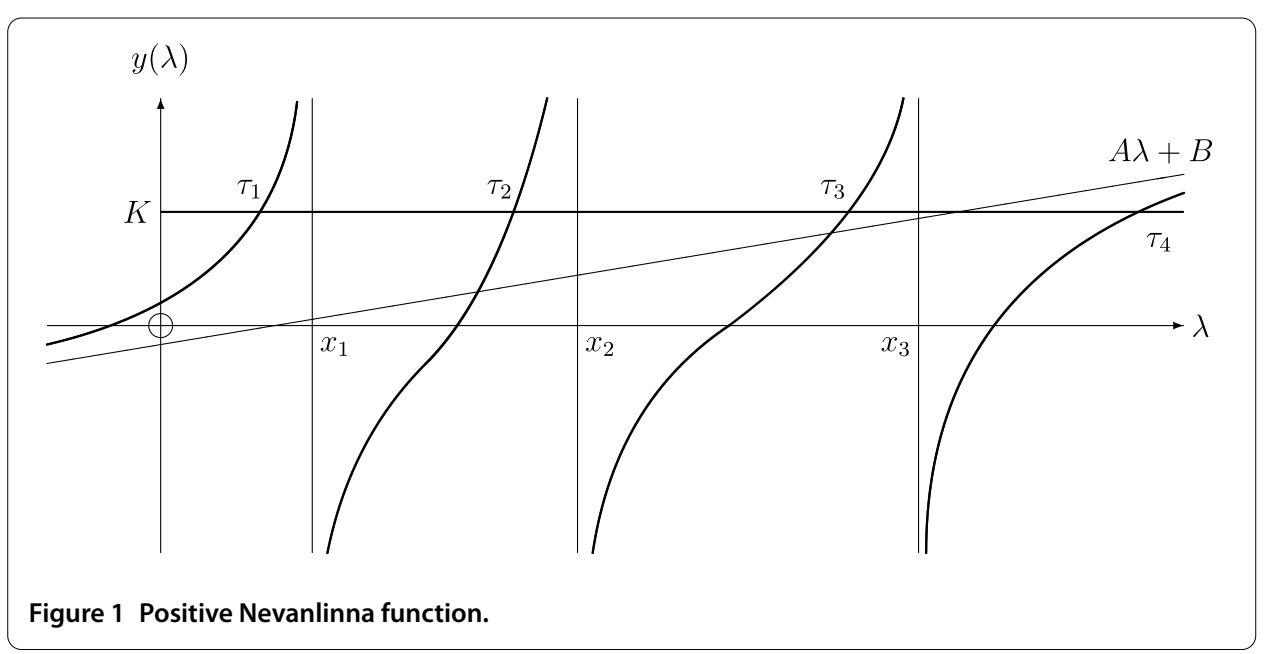

$j$ indicates the value of $n$ at which the boundary condition is imposed and

$$
\diamond= \begin{cases} \pm & \text { if the coefficient of } \lambda \text { is positive or negative resp. } \\ 0 & \text { if the coefficient of } \lambda \text { is zero. }\end{cases}
$$

The number of eigenvalues that a particular boundary value problem has depends on the form of the boundary conditions and the theorem below was proved in [4].

Theorem 2.2 Consider the boundary value problem given by equation (1.1) for $n=$ $0, \ldots, r-1$, together with boundary conditions

$$
\begin{aligned}
& y(-1)=\left[a \lambda+b-\sum_{k=1}^{p} \frac{c_{k}}{\lambda-d_{k}}\right] y(0), \quad a \geq 0, c_{k}>0 ; \\
& y(r-1)=\left[\alpha \lambda+\beta-\sum_{j=1}^{s} \frac{\gamma_{j}}{\lambda-\sigma_{j}}\right] y(r), \quad \alpha \leq 0, \gamma_{j}<0 .
\end{aligned}
$$

Then boundary value problem (1.1), (2.9), (2.10) has

(i) $s+p+r+1$ eigenvalues if $\alpha<0$,

(ii) $s+p+r$ eigenvalues if $\alpha=0$ and $\beta \neq 0$,

(iii) $s+p+r-1$ eigenvalues if $\alpha=\beta=0$.

(Note that the number of unit intervals considered is $r+1$.)

\section{Transformation of boundary conditions}

In this section we investigate how $y(n)$ obeying general eigenparameter-dependent boundary conditions of the form given in (1.2) and (1.3) transforms under (2.1) to $v(n)$ obeying corresponding boundary conditions which depend on the spectral parameter. Choosing various zero or non-zero values for $a, b, g, h$ gives rise to the different results which are proved below. By considering the number of zero's and poles (singularities) of the various Nevanlinna functions involved, we obtain the precise form of these transformed boundary conditions. 


\subsection{Boundary condition at the terminal end point}

The following lemma could be considered as an analogy to Lemma 3.1 in [2].

Lemma 3.1 If $y(n)$ obeys the boundary condition

$$
\begin{aligned}
y(m-1) & =\left[a \lambda+b-\sum_{k=1}^{s} \frac{c_{k}}{\lambda-d_{k}}\right] y(m), \quad c_{k}<0 \\
& :=R_{s, m}^{\diamond}(\lambda) y(m) \quad \text { for } \diamond= \begin{cases}- & \text { if } a<0, \\
0 & \text { if } a=0,\end{cases}
\end{aligned}
$$

then the domain of $v(n)$ may be extended from $n=-1, \ldots, m-1$ to $n=-1, \ldots, m$ by forcing the condition

$$
\frac{v(m-1)}{v(m)}=\frac{1}{W}
$$

where

$$
W=\frac{c(m-1) z(m-1)}{c(m) z(m)}-\lambda+\frac{\frac{z(m) \lambda}{z(m-1)}}{\frac{z(m)}{z(m-1)}-\frac{1}{R_{s, m}^{\circ}(\lambda)}} .
$$

Proof The transformed equation, (2.2), for $n=m-1$, together with (3.2) gives

$$
\begin{aligned}
& c_{v}(m-1) W v(m-1)-b_{v}(m-1) v(m-1)+c_{\nu}(m-2) v(m-2) \\
& \quad=-\lambda c_{v}(m-1) v(m-1) .
\end{aligned}
$$

From mapping (2.1), with $n=m-1$, and boundary condition (3.1), we obtain

$$
v(m-1)=y(m)\left[1-R_{s, m}^{\diamond}(\lambda) \frac{z(m)}{z(m-1)}\right] .
$$

Similarly, (2.1) with $n=m-2$ and (3.1) yields

$$
v(m-2)=R_{s, m}^{\diamond}(\lambda) y(m)-y(m-2) \frac{z(m-1)}{z(m-2)} .
$$

Rearranging equation (1.1) with $n=m-1$ gives

$$
c(m-2) y(m-2)=b(m-1) y(m-1)-\lambda c(m-1) y(m-1)-c(m-1) y(m) .
$$

Solving for $y(m-2)$ in (3.7) and substituting this result into (3.6) together with boundary condition (3.1), we have that

$$
\begin{aligned}
v(m-2)= & y(m)\left[R_{s, m}^{\diamond}(\lambda)\right. \\
& \left.-\frac{z(m-1)}{c(m-2) z(m-2)}\left([b(m-1)-\lambda c(m-1)] R_{s, m}^{\diamond}(\lambda)-c(m-1)\right)\right] .
\end{aligned}
$$


Now substitute (3.5) and (3.8) into (3.4) and divide through by $y(m) \neq 0$ to obtain

$$
\begin{aligned}
W= & \frac{b_{\nu}(m-1)-\lambda c_{\nu}(m-1)}{c_{\nu}(m-1)}-\frac{c_{\nu}(m-2)}{c_{\nu}(m-1)} \\
& \times \frac{\left[R_{s, m}^{\diamond}(\lambda)-\frac{z(m-1)}{c(m-2) z(m-2)}\left(R_{s, m}^{\diamond}(\lambda)[b(m-1)-\lambda c(m-1)]-c(m-1)\right)\right]}{1-\frac{z(m)}{z(m-1)} R_{s, m}^{\diamond}(\lambda)} .
\end{aligned}
$$

The above equation may be simplified as follows. First, divide the numerator and denominator of the second term on the right-hand side by $R_{s, m}^{\diamond}(\lambda) \neq 0$ to get

$$
\begin{aligned}
W= & \frac{b_{v}(m-1)}{c_{\nu}(m-1)}-\lambda \\
& -\frac{c_{\nu}(m-2)}{c_{\nu}(m-1)}\left[\frac{1-\frac{z(m-1)}{c(m-2) z(m-2)}\left(b(m-1)-\lambda c(m-1)-\frac{c(m-1)}{R_{s, m}^{s}(\lambda)}\right)}{\frac{1}{R_{s, m}(\lambda)}-\frac{z(m)}{z(m-1)}}\right] .
\end{aligned}
$$

Since $z(n)$ obeys (1.1) for $\lambda=0$ with $n=m-1$, we obtain

$$
1-\frac{b(m-1) z(m-1)}{c(m-2) z(m-2)}=-\frac{c(m-1) z(m)}{c(m-2) z(m-2)} .
$$

If we substitute (3.11) into the numerator of the term in square brackets in (3.10), then the numerator becomes

$$
\frac{c(m-1) z(m-1)}{c(m-2) z(m-2)}\left(\lambda+\frac{1}{R_{s, m}^{\diamond}(\lambda)}-\frac{z(m)}{z(m-1)}\right),
$$

and so the ratio contained in the square brackets simplifies to

$$
\frac{\frac{c(m-1) z(m-1)}{c(m-2) z(m-2)} \lambda}{\frac{1}{R_{s, m}^{0}(\lambda)}-\frac{z(m)}{z(m-1)}}+\frac{c(m-1) z(m-1)}{c(m-2) z(m-2)} .
$$

Substitution of (3.13) into equation (3.10) results in the following expression for $W$ :

$$
W=\frac{b_{\nu}(m-1)}{c_{\nu}(m-1)}-\lambda-\frac{c_{\nu}(m-2)}{c_{\nu}(m-1)} \frac{c(m-1) z(m-1)}{c(m-2) z(m-2)}-\frac{\frac{c_{v}(m-2) c(m-1) z(m-1)}{c_{\nu}(m-1) c(m-2) z(m-2)} \lambda}{\frac{1}{R_{s, m}(\lambda)}-\frac{z(m)}{z(m-1)}} .
$$

From (2.3),

$$
c_{v}(n)=\frac{c(n) z(n)}{z(n+1)}
$$

thus

$$
\frac{c_{v}(m-2)}{c_{v}(m-1)}=\frac{c(m-2) z(m-2)}{z(m-1)} \frac{z(m)}{c(m-1) z(m-1)},
$$

and hence a simplified expression for $W$ is given by

$$
W=\frac{b_{v}(m-1)}{c_{v}(m-1)}-\lambda-\frac{z(m)}{z(m-1)}+\frac{\frac{z(m)}{z(m-1)} \lambda}{\frac{z(m)}{z(m-1)}-\frac{1}{R_{s, m}^{\dagger}(\lambda)}} .
$$


Also from (2.4), with $n=m-1$, we obtain

$$
b_{v}(m-1)=\left[\frac{c(m-1) z(m-1)}{c(m) z(m)}+\frac{z(m)}{z(m-1)}\right] \frac{c(m-1) z(m-1)}{z(m)} .
$$

Thus the first term on the right-hand side of (3.16) becomes

$$
\frac{b_{v}(m-1)}{c_{v}(m-1)}=\frac{c(m-1) z(m-1)}{c(m) z(m)}+\frac{z(m)}{z(m-1)}
$$

and hence

$$
W=\frac{c(m-1) z(m-1)}{c(m) z(m)}-\lambda+\frac{\frac{z(m)}{z(m-1)} \lambda}{\frac{z(m)}{z(m-1)}-\frac{1}{R_{s, m}^{\diamond}(\lambda)}}
$$

as required.

It is still necessary to show that $1 / W$ is a negative Nevanlinna function of the correct form. In the following theorem, we consider the case where $a=0$ and $b \neq 0$ in (3.1).

Theorem 3.2 Consider $y(n)$ obeying the boundary condition

$$
\begin{aligned}
y(m-1) & =\left[b-\sum_{k=1}^{s} \frac{c_{k}}{\lambda-d_{k}}\right] y(m), \quad c_{k}<0,0<\frac{1}{b}<\frac{z(m)}{z(m-1)} \\
& :=R_{s, m}^{0}(\lambda) y(m),
\end{aligned}
$$

then $y(n)$ transforms, under (2.1), to $v(n)$ obeying (3.2) as follows:

(1) If $z(n)$ does not obey (3.20) for $\lambda=0$, then $v(n)$ obeys

$$
v(m-1)=\frac{1}{W} v(m)=\left[-\sum_{j=1}^{s+1} \frac{\sigma_{j}}{\lambda-\delta_{j}}\right] v(m):=T_{s+1, m}^{0}(\lambda) v(m) .
$$

(2) If $z(n)$ does obey (3.20), then $v(n)$ obeys

$$
v(m-1)=\frac{1}{W} v(m)=\left[-\sum_{j=1}^{s} \frac{\tilde{\sigma}_{j}}{\lambda-\tilde{\delta}_{j}}\right] v(m):=\tilde{T}_{s, m}^{0}(\lambda) v(m),
$$

where $\sigma_{j}, \tilde{\sigma}_{j}<0$.

Proof Recall from Nevanlinna property (A) that if $R_{s, m}^{0}(\lambda)$ is a negative Nevanlinna function, then $\frac{1}{R_{s, m}^{0}(\lambda)}$ is a positive Nevanlinna function. To show that $\frac{1}{W}$ is a negative Nevanlinna function, where $W$ is as given in (3.3) with $\diamond=0$, we consider the ratio

$$
\frac{\lambda}{\frac{z(m)}{z(m-1)}-\frac{1}{R_{s, m}^{0}(\lambda)}}
$$


which has expansion

$$
f(\lambda)-\sum_{t=1}^{p} \frac{r_{t}}{\lambda-q_{t}}, \quad r_{t}>0
$$

where $q_{t}$ 's correspond to $\frac{1}{R_{s, m}^{0}(\lambda)}=\frac{z(m)}{z(m-1)}$, i.e. the singularities of (3.23). By Nevanlinna property (B),

$$
\frac{1}{R_{s, m}^{0}(\lambda)}=\frac{1}{b}-\sum_{j=1}^{s} \frac{\sigma_{j}}{\lambda-\delta_{j}}, \quad \sigma_{j}>0
$$

Since $\frac{1}{R_{s, m}^{0}(\lambda)}$ is a positive Nevanlinna function, it has a graph of the form shown in Figure 1 where $y(\lambda)=\frac{1}{R_{s, m}^{0}(\lambda)}, K=\frac{z(m)}{z(m-1)}, A=0, B=\frac{1}{b}$ such that $0<B<K, x_{i}=\delta_{i}$ and $\tau_{i}=q_{i}$ for $i=1,2,3$. Note that since $A=0, \tau_{4}$ does not exist.

Clearly, the gradient of $\frac{1}{R_{s, m}^{0}(\lambda)}$ at $q_{t}$ is positive for all $t$, that is,

$$
\left.\frac{\partial}{\partial \lambda} \frac{1}{R_{s, m}^{0}(\lambda)}\right|_{q t}>0, \quad t=1, \ldots, p
$$

If $z(n)$ does not obey (3.20), then the zeros of $\frac{\lambda}{\frac{z(m)}{z(m-1)}-\frac{1}{R_{s, m}^{0}(\lambda)}}$ are the poles of $\frac{1}{R_{s, m}^{0}(\lambda)}$, that is, $\delta_{j}$ 's together with $\lambda=0$. Since there is the same number of $q_{t}$ 's as there are $\delta_{j}$ 's, it follows that $p=s$ in (3.24).

We now examine the form of $f(\lambda)$ in (3.24) more closely. Using (3.25) we may write

$$
\frac{1}{R_{s, m}^{0}(\lambda)}=\frac{\frac{1}{b} \prod_{j=1}^{s}\left(\lambda-\delta_{j}\right)-\sum_{i=1}^{s} \prod_{j=1, j \neq i}^{s} \sigma_{j}\left(\lambda-\delta_{j}\right)}{\prod_{j=1}^{s}\left(\lambda-\delta_{j}\right)} .
$$

Thus it follows that

$$
\begin{aligned}
\frac{\lambda}{\frac{z(m)}{z(m-1)}-\frac{1}{R_{s, m}^{0}(\lambda)}} & =\frac{\lambda \prod_{j=1}^{s}\left(\lambda-\delta_{j}\right)}{\frac{z(m)}{z(m-1)} \prod_{j=1}^{s}\left(\lambda-\delta_{j}\right)-\frac{1}{b} \prod_{j=1}^{s}\left(\lambda-\delta_{j}\right)+\sum_{i=1}^{s} \prod_{j=1, j \neq i}^{s} \sigma_{j}\left(\lambda-\delta_{j}\right)} \\
& :=\frac{\lambda^{s+1}+a_{s} \lambda^{s}+a_{s-1} \lambda^{s-1}+\cdots+a_{1} \lambda}{\left(\frac{z(m)}{z(m-1)}-\frac{1}{b}\right) \lambda^{s}+b_{s-1} \lambda^{s-1}+\cdots+b_{0}} \\
& :=\frac{1}{\frac{z(m)}{z(m-1)}-\frac{1}{b}} \lambda+C+\frac{c_{s-1} \lambda^{s-1}+\cdots+c_{0}}{\left(\frac{z(m)}{z(m-1)}-\frac{1}{b}\right) \lambda^{s}+b_{s-1} \lambda^{s-1}+\cdots+b_{0}} \\
& \rightarrow \frac{1}{\frac{z(m)}{z(m-1)}-\frac{1}{b}} \lambda+C
\end{aligned}
$$

as $\lambda \rightarrow \pm \infty$, where $C, a_{i}, b_{j}$ and $c_{j}$ are constants for $i=1, \ldots, s$ and $j=0, \ldots, s-1$. It should be noted that the exact form of $C$ is not required at this stage. Therefore, $f(\lambda)=\frac{\lambda}{\frac{z(m)}{z(m-1)}-\frac{1}{b}}+C$. 
This together with (3.23), (3.24) and (3.3) gives

$$
\begin{aligned}
W & =\frac{c(m-1) z(m-1)}{c(m) z(m)}-\lambda+\frac{z(m)}{z(m-1)}\left[\frac{\lambda}{\frac{z(m)}{z(m-1)}-\frac{1}{b}}+C-\sum_{t=1}^{s} \frac{r_{t}}{\lambda-q_{t}}\right] \\
& :=\Gamma \lambda+\Omega-\sum_{t=1}^{s} \frac{\gamma r_{t}}{\lambda-q_{t}},
\end{aligned}
$$

where $\gamma=\frac{z(m)}{z(m-1)}, \Gamma=\frac{\gamma}{\gamma-\frac{1}{b}}-1$ and $\Omega=\frac{c(m-1)}{c(m)} \gamma^{-1}+C \gamma$. Since $\gamma r_{t}>0$, it follows that $W$ can only be a positive Nevanlinna function if $\Gamma>0$, which implies that

$$
\frac{\frac{z(m)}{z(m-1)}}{\frac{z(m)}{z(m-1)}-\frac{1}{b}}-1>0 \quad \Rightarrow \quad \frac{\frac{1}{b}}{\frac{z(m)}{z(m-1)}-\frac{1}{b}}>0 .
$$

This results in two cases: either $\frac{1}{b}<0$ and $\frac{z(m)}{z(m-1)}<\frac{1}{b}$, which is not possible as $\frac{z(m)}{z(m-1)}>0$, or we must have $\frac{1}{b}>0$ and $\frac{z(m)}{z(m-1)}>\frac{1}{b}$. This in turn means that for $0<\frac{1}{b}<\frac{z(m)}{z(m-1)}$ it follows by Nevanlinna property $(C)$ that $\frac{1}{W}$ is a negative Nevanlinna function of the form (3.21) as required.

If $z(n)$ does obey (3.20) for $\lambda=0$, then $\frac{z(m-1)}{z(m)}=R_{s, m}^{0}(0)$, i.e. $\frac{1}{R_{s, m}^{0}(0)}=\frac{z(m)}{z(m-1)}$. Thus, one of $q_{t}$ 's, $t=1, \ldots, p$, is equal to 0 .

Now, since $\frac{1}{R_{s, m}^{0}(0)}=\frac{z(m)}{z(m-1)}$, using (3.25), routine calculations give

$$
\frac{\lambda}{\frac{z(m)}{z(m-1)}-\frac{1}{R_{s, m}^{0}(\lambda)}}=\frac{1}{\sum_{j=1}^{S} \frac{\sigma_{j}}{\delta_{j}\left(\lambda-\delta_{j}\right)}},
$$

which illustrates that the discontinuity at $\lambda=0$ is removable. So, the number of nonremovable $q_{t}$ 's is one less than the number of $\delta_{j}$ 's. This means that the number of terms in (3.24) is $s-1$ and since we may relabel, if required, we take $p=s-1$. Therefore, in the same manner as above,

$$
\begin{aligned}
W & =\frac{c(m-1) z(m-1)}{c(m) z(m)}-\lambda+\frac{z(m)}{z(m-1)}\left[\frac{\lambda}{\frac{z(m)}{z(m-1)}-\frac{1}{b}}+C-\sum_{t=1}^{s-1} \frac{r_{t}}{\lambda-q_{t}}\right] \\
& :=\Gamma \lambda+\Omega-\sum_{t=1}^{s-1} \frac{\gamma r_{t}}{\lambda-q_{t}},
\end{aligned}
$$

where $\gamma, \Gamma$ and $\Omega$ are as previously defined. Once again, for $W$ to be a positive Nevanlinna function, we require that $0<\frac{1}{b}<\frac{z(m)}{z(m-1)}$. Hence, by Nevanlinna result $(C), \frac{1}{W}$ may be written as a negative Nevanlinna function of the form (3.22).

Theorem 3.3 Consider $y(n)$ obeying the boundary condition

$$
\begin{aligned}
y(m-1) & =\left[a \lambda+b-\sum_{k=1}^{s} \frac{c_{k}}{\lambda-d_{k}}\right] y(m), \quad a<0, c_{k}<0 \text { and } \frac{z(m-1)}{z(m)}<b \\
& :=R_{s, m}^{-}(\lambda) y(m) .
\end{aligned}
$$

Under mapping (2.1), $y(n)$ obeying (3.28) transforms to $v(n)$ obeying the following: 
(A) If $z(n)$ does not obey (3.28) for $\lambda=0$, then $v(n)$ obeys a boundary condition of the form

(i)

$$
\begin{aligned}
v(m-1) & =\frac{1}{W} v(m)=\left[\alpha \lambda+\beta-\sum_{j=1}^{s} \frac{\eta_{j}}{\lambda-\epsilon_{j}}\right] v(m), \quad a=\frac{-c(m)}{c(m-1)} \\
& :=T_{s, m}^{-}(\lambda) v(m) ;
\end{aligned}
$$

(ii)

$$
\begin{aligned}
v(m-1) & =\frac{1}{W} v(m)=\left[\tilde{\beta}-\sum_{j=1}^{s+1} \frac{\tilde{\eta}_{j}}{\lambda-\tilde{\epsilon}_{j}}\right] v(m), \quad a \neq \frac{-c(m)}{c(m-1)} \\
& :=\tilde{T}_{s+1, m}^{0}(\lambda) \nu(m) .
\end{aligned}
$$

(B) If $z(n)$ does obey (3.28) for $\lambda=0$, then $v(n)$ obeys a boundary condition of the form

(i)

$$
\begin{aligned}
v(m-1) & =\frac{1}{W} v(m)=\left[\hat{\alpha} \lambda+\hat{\beta}-\sum_{j=1}^{s-1} \frac{\hat{\eta}_{j}}{\lambda-\hat{\epsilon}_{j}}\right] v(m), \quad a=\frac{-c(m)}{c(m-1)} \\
& :=\hat{T}_{s-1, m}^{-}(\lambda) v(m) ;
\end{aligned}
$$

(ii)

$$
\begin{aligned}
v(m-1) & =\frac{1}{W} v(m)=\left[\bar{\beta}-\sum_{j=1}^{s} \frac{\bar{\eta}_{j}}{\lambda-\bar{\epsilon}_{j}}\right] v(m), \quad a \neq \frac{-c(m)}{c(m-1)} \\
& :=\bar{T}_{s, m}^{0}(\lambda) v(m),
\end{aligned}
$$

where $\alpha, \hat{\alpha}, \eta_{j}, \tilde{\eta}_{j}, \hat{\eta}_{j}, \bar{\eta}_{j}<0$, i.e. $T_{s, m}^{-}(\lambda), \tilde{T}_{s+1, m}^{0}(\lambda), \hat{T}_{s-1, m}^{-}(\lambda), \bar{T}_{s, m}^{0}(\lambda)$ are negative Nevanlinna functions.

Proof Since $R_{s, m}^{-}(\lambda)$ is a negative Nevanlinna function, it follows by Nevanlinna property (A) that $\frac{1}{R_{s, m}^{-}(\lambda)}$ is a positive Nevanlinna function of the form

$$
\frac{1}{R_{s, m}^{-}(\lambda)}=-\sum_{j=1}^{s+1} \frac{\tilde{\sigma}_{j}}{\lambda-\tilde{\delta}_{j}}, \quad \tilde{\sigma}_{j}>0 .
$$

To demonstrate that $\frac{1}{W}$ is a negative Nevanlinna function, we consider the ratio

$$
\frac{\lambda}{\frac{z(m)}{z(m-1)}-\frac{1}{R_{s, m}^{-}(\lambda)}}
$$

which has expansion

$$
\tilde{f}(\lambda)=\sum_{t=1}^{p} \frac{\tilde{r}_{t}}{\lambda-\tilde{q}_{t}}
$$


where $\tilde{r}_{t}>0$. Here $\tilde{q}_{t}$ 's correspond to $\frac{z(m)}{z(m-1)}=\frac{1}{R_{s, m}(\lambda)}$ which are the poles of $\frac{1}{R_{s, m}^{s}(\lambda)}\left(\right.$ i.e. $\tilde{\delta}_{j}$ 's $)$ and $\lambda=0$.

The graph of $\frac{1}{\overline{R_{s, m}(\lambda)}}$ is given by Figure 1 with $y(\lambda)=\frac{1}{R_{s, m}^{-}(\lambda)}, K=\frac{z(m)}{z(m-1)}, A=B=0, x_{i}=\tilde{\delta}_{i}$ and $\tau_{i}=\tilde{q}_{i}$ for $i=1,2,3$. Note again that since $A=0, \tau_{4}$ does not exist.

Clearly, the gradient of $\frac{1}{R_{s, m}(\lambda)}$ at $\tilde{q}_{t}$ is positive and the number of $\tilde{q}_{t}$ 's is the same as the number of $\tilde{\delta}_{j}$ 's, thus in (3.35), $p=s+1$.

As $\lambda \rightarrow \pm \infty$, it follows that

$$
\frac{1}{R_{s, m}^{-}(\lambda)} \rightarrow \frac{1}{a \lambda+b}
$$

and hence

$$
\tilde{f}(\lambda)-\sum_{t=1}^{s+1} \frac{\tilde{r}_{t}}{\lambda-\tilde{q}_{t}}=\frac{\lambda}{\frac{z(m)}{z(m-1)}-\frac{1}{R_{s, m}^{-}(\lambda)}} \rightarrow \frac{\lambda}{\frac{z(m)}{z(m-1)}-\frac{1}{(a \lambda+b)}}=c_{1} \lambda+c_{2}+\frac{-\frac{c_{3}}{a z(m)}}{\lambda+\frac{b}{a}-\frac{z(m-1)}{a z(m)}},
$$

where $c_{1}=\frac{z(m-1)}{z(m)}, c_{2}=\frac{z(m-1)^{2}}{a z(m)^{2}}$ and $c_{3}=\frac{z(m-1)^{2}}{a z(m)} b-\frac{z(m-1)^{3}}{a z(m)^{2}}<0$. Since $a<0$, it follows that $\frac{c_{3}}{a z(m)}>0$.

Thus $\tilde{f}(\lambda)=c_{1} \lambda+c_{2}$ and therefore

$$
\begin{aligned}
W & =\frac{c(m-1) z(m-1)}{c(m) z(m)}-\lambda+\frac{z(m)}{z(m-1)}\left[\tilde{f}(\lambda)-\sum_{t=1}^{s+1} \frac{\tilde{r}_{t}}{\lambda-\tilde{q}_{t}}\right] \\
& =\frac{c(m-1) z(m-1)}{c(m) z(m)}+\frac{z(m-1)}{a z(m)}-\sum_{t=1}^{s+1} \frac{z(m)}{\frac{z(m-1)}{z_{t}}} \tilde{r}_{t}
\end{aligned}
$$

If $\frac{z(m-1)}{z(m)}\left[\frac{c(m-1)}{c(m)}+\frac{1}{a}\right]=0$, it implies that $a=\frac{-c(m)}{c(m-1)}$, and then

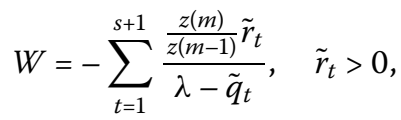

which is a positive Nevanlinna function. So, by Nevanlinna property $(C)$,

$$
\frac{1}{W}=\alpha \lambda+\beta-\sum_{j=1}^{s} \frac{\eta_{j}}{\lambda-\epsilon_{j}}, \quad \alpha<0, \eta_{j}<0
$$

as required by (3.29).

$$
\text { If } a \neq \frac{-c(m)}{c(m-1)} \text {, then for } b>\frac{z(m-1)}{z(m)} \text { we have }
$$

$$
W=\frac{1}{\tilde{\beta}}-\sum_{t=1}^{s+1} \frac{z(m)}{z(m-1)} \tilde{r}_{t}, \quad \tilde{r}_{t}>0,
$$

where $\frac{1}{\tilde{\beta}}=\frac{c(m-1) z(m-1)}{c(m) z(m)}+\frac{z(m-1)}{a z(m)} \neq 0$. By Nevanlinna property (B), we obtain

$$
\frac{1}{W}=\tilde{\beta}-\sum_{j=1}^{s+1} \frac{\tilde{\eta}_{j}}{\lambda-\tilde{\epsilon}_{j}}, \quad \tilde{\eta}_{j}<0,
$$

that is, (3.30) holds. 
If $z(n)$ does obey (3.28) for $\lambda=0$, then $\frac{z(m-1)}{z(m)}=R_{s, m}^{-}(0)$, that is, $\frac{1}{R_{s, m}^{-}(0)}=\frac{z(m)}{z(m-1)}$. This implies that one of $\tilde{q}_{t}$ 's, $t=1, \ldots, p$, is equal to 0 . Thus, as in Theorem 3.2,

$$
\frac{\lambda}{\frac{z(m)}{z(m-1)}-\frac{1}{R_{s, m}^{-}(\lambda)}}=\frac{1}{\sum_{j=1}^{s+1} \frac{\tilde{\sigma}_{j} / \tilde{\delta}_{j}}{\lambda-\tilde{\delta}_{j}}} .
$$

Therefore, the discontinuity at $\lambda=0$ is removable giving that the number of nonremovable $\tilde{q}_{t}$ 's is one less than the number of $\tilde{\delta}_{j}$ 's. This implies that in (3.35) the number of terms in the sum equals $s$ so, relabeling if necessary, we can set $p=s$.

The remainder of the results are obtained in exactly the same manner as in the case where $z(n)$ does not obey boundary condition (3.28) with $s$ being replaced by $s-1$, see (3.36) and the subsequent calculations. Thus we obtain the following:

$$
\begin{gathered}
\text { If } a=\frac{-c(m)}{c(m-1)} \text {, then for } b>\frac{z(m-1)}{z(m)} \text { we have } \\
W=-\sum_{t=1}^{s} \frac{\frac{1}{R_{s, m}^{-}(0)} \tilde{r}_{t}}{\lambda-\tilde{q}_{t}}, \quad \tilde{r}_{t}>0,
\end{gathered}
$$

and hence, by Nevanlinna property $(\mathrm{C})$,

$$
\frac{1}{W}=\hat{\alpha} \lambda+\hat{\beta}-\sum_{j=1}^{s-1} \frac{\hat{\eta}_{j}}{\lambda-\hat{\epsilon}_{j}}, \quad \hat{\alpha}<0, \hat{\eta}_{j}<0,
$$

which is a negative Nevanlinna function of the correct form, i.e. equation (3.31).

If $a \neq \frac{-c(m)}{c(m-1)}$, then for $b>\frac{z(m-1)}{z(m)}$ we have

$$
W=\frac{1}{\bar{\beta}}-\sum_{t=1}^{s} \frac{\frac{1}{R_{s, m}^{-}(0)} \tilde{r}_{t}}{\lambda-\tilde{q}_{t}}, \quad \frac{1}{\bar{\beta}} \neq 0, \tilde{r}_{t}>0
$$

and hence

$$
\frac{1}{W}=\bar{\beta}-\sum_{j=1}^{s} \frac{\bar{\eta}_{j}}{\lambda-\bar{\epsilon}_{j}}, \quad \bar{\eta}_{j}<0
$$

where $\frac{1}{\bar{\beta}}=\frac{c(m-1)}{c(m)} R_{s, m}^{-}(0)+\frac{R_{s, m}^{-}(0)}{a}$. That is, we obtain (3.32).

Theorem 3.4 Consider the boundary condition

$$
\begin{aligned}
y(m-1) & =\left[-\sum_{k=1}^{s} \frac{c_{k}}{\lambda-d_{k}}\right] y(m), \quad c_{k}<0 \\
& :=R_{s, m}^{0}(\lambda) y(m) .
\end{aligned}
$$

Under mapping (2.1), $y(n)$ obeying (3.37) transforms to $v(n)$ obeying boundary conditions of the following form:

(A) If $z(n)$ does not obey (3.37) for $\lambda=0$, then $v(n)$ obeys a boundary condition of the form

$$
v(m-2)=\left[\phi-\sum_{t=1}^{s} \frac{h_{t}}{\lambda-g_{t}}\right] v(m-1):=U_{s, m-1}^{0}(\lambda) v(m-1) .
$$


(B) If $z(n)$ does obey (3.37) for $\lambda=0$, then $v(n)$ obeys a boundary condition of the form

$$
v(m-2)=\left[\phi-\sum_{t=1}^{s-1} \frac{\tilde{h}_{t}}{\lambda-\tilde{g}_{t}}\right] v(m-1):=\tilde{U}_{s-1, m-1}^{0}(\lambda) v(m-1),
$$

where $h_{t}, \tilde{h}_{t}<0$, i.e. $U_{s, m-1}^{0}(\lambda), \tilde{U}_{s-1, m-1}^{0}(\lambda)$ are negative Nevanlinna functions.

Proof Using (2.1) with $n=m-2$ and $n=m-1$ gives, respectively,

$$
v(m-2)=y(m-1)-y(m-2) \frac{z(m-1)}{z(m-2)}
$$

and

$$
v(m-1)=y(m)-y(m-1) \frac{z(m)}{z(m-1)}=\left[1-\frac{z(m)}{z(m-1)} R_{s, m}^{0}(\lambda)\right] y(m)
$$

by using (3.37). Now, (1.1) evaluated at $n=m-1$ gives

$$
c(m-1) y(m)-b(m-1) y(m-1)+c(m-2) y(m-2)=-\lambda c(m-1) y(m-1) .
$$

Using (3.37) and dividing through by $c(m-2)$ yields

$$
y(m-2)=\frac{1}{c(m-2)}\left([b(m-1)-\lambda c(m-1)] R_{s, m}^{0}(\lambda)-c(m-1)\right) y(m) .
$$

Substitute (3.42) into (3.40) and use (3.37) to obtain

$$
\begin{aligned}
v(m-2)= & \left\{R_{s, m}^{0}(\lambda)-\frac{z(m-1)}{c(m-2) z(m-2)}\right. \\
& \left.\times\left[(b(m-1)-\lambda c(m-1)) R_{s, m}^{0}(\lambda)-c(m-1)\right]\right\} y(m) .
\end{aligned}
$$

From equations (3.41) and (3.43), we have the condition

$$
\frac{v(m-2)}{v(m-1)}=\frac{1-\frac{z(m-1)}{c(m-2) z(m-2)}\left[b(m-1)-\lambda c(m-1)-c(m-1) \frac{1}{R_{s, m}^{0}(\lambda)}\right]}{\frac{1}{R_{s, m}^{0}(\lambda)}-\frac{z(m)}{z(m-1)}} .
$$

But $z(n)$ obeys (1.1) for $\lambda=0$, so with $n=m-1$ and after division by $c(m-2) z(m-2)$, one gets

$$
1-\frac{b(m-1) z(m-1)}{c(m-2) z(m-2)}=-\frac{c(m-1) z(m)}{c(m-2) z(m-2)} .
$$

The substitution of (3.45) into (3.44) results in

$$
\begin{aligned}
\frac{v(m-2)}{v(m-1)} & =\frac{\frac{-c(m-1) z(m)}{c(m-2) z(m-2)}+\frac{\lambda c(m-1) z(m-1)}{c(m-2) z(m-2)}+\frac{c(m-1) z(m-1)}{c(m-2) z(m-2)} \frac{1}{R_{s, m}^{0}(\lambda)}}{\frac{1}{R_{s, m}^{0}(\lambda)}-\frac{z(m)}{z(m-1)}} \\
& =\frac{c(m-1) z(m-1)}{c(m-2) z(m-2)}-\frac{\frac{\lambda c(m-1) z(m-1)}{c(m-2) z(m-2)}}{\frac{z(m)}{z(m-1)}-\frac{1}{R_{s, m}^{0}(\lambda)}} .
\end{aligned}
$$


Now,

$$
\frac{\lambda}{\frac{z(m)}{z(m-1)}-\frac{1}{R_{s, m}^{0}(\lambda)}}
$$

has the expansion

$$
\hat{f}(\lambda)-\sum_{t=1}^{p} \frac{\hat{r}_{t}}{\lambda-\hat{q}_{t}}, \quad \hat{r}_{t}>0
$$

where $\hat{q}_{t}$ 's correspond to $\frac{1}{R_{s, m}^{0}(\lambda)}=\frac{z(m)}{z(m-1)}$, i.e. the singularities of (3.46).

Recall from (3.37) that

$$
R_{s, m}^{0}(\lambda)=-\sum_{k=1}^{s} \frac{c_{k}}{\lambda-d_{k}}, \quad c_{k}<0
$$

By Nevanlinna property $(C)$ we have that

$$
\frac{1}{R_{s, m}^{0}(\lambda)}=\omega \lambda+\xi-\sum_{k=1}^{s-1} \frac{\mu_{k}}{\lambda-\rho_{k}}, \quad \omega>0, \mu_{k}>0
$$

As $\frac{1}{R_{s, m}^{0}(\lambda)}$ is a positive Nevanlinna function, it has a graph of the form shown in Figure 1 where $y(\lambda)=\frac{1}{R_{s, m}^{0}(\lambda)}, K=\frac{z(m)}{z(m-1)}, A=\omega, B=\xi, x_{j}=\rho_{j}$ for $j=1,2,3$ and $\tau_{i}=\hat{q}_{i}$ for $i=1,2,3,4$.

Observe that the gradient of $\frac{1}{R_{s, m}^{0}(\lambda)}$ at $\hat{q}_{t}$ is positive for all $t=1, \ldots, p$.

If $z(n)$ does not obey (3.37) for $\lambda=0$, then the zeros of

$$
\frac{\lambda}{\frac{z(m)}{z(m-1)}-\frac{1}{R_{s, m}^{0}(\lambda)}}
$$

are the poles of $\frac{1}{R_{s, m}^{0}(\lambda)}\left(\right.$ i.e. $\rho_{k}$ 's) and $\lambda=0$. It is evident that the number of $\hat{q}_{t}$ 's is one more than the number of $\rho_{k}$ 's, thus in (3.47), $p=s$.

Next, if we let $\lambda \rightarrow \pm \infty$, it follows that $\frac{1}{R_{s, m}^{0}(\lambda)} \rightarrow \omega \lambda+\xi$. Examining the behavior of (3.47), one sees that

$$
\frac{\lambda}{\frac{z(m)}{z(m-1)}-\frac{1}{R_{s, m}^{0}(\lambda)}} \rightarrow \frac{\lambda}{\frac{z(m)}{z(m-1)}-(\omega \lambda+\xi)} \rightarrow-\frac{1}{\omega}=\hat{f}(\lambda) .
$$

Hence

$$
\begin{aligned}
\frac{v(m-2)}{v(m-1)} & =\frac{c(m-1) z(m-1)}{c(m-2) z(m-2)}-\left[\hat{f}(\lambda)-\sum_{t=1}^{s} \frac{\hat{r}_{t}}{\lambda-\hat{q}_{t}}\right] \frac{c(m-1) z(m-1)}{c(m-2) z(m-2)}, \quad \hat{r}_{t}>0 \\
& :=\phi-\sum_{t=1}^{s} \frac{h_{t}}{\lambda-g_{t}},
\end{aligned}
$$

where $\phi=\frac{c(m-1) z(m-1)}{c(m-2) z(m-2)}\left(1+\frac{1}{\omega}\right), g_{t}=\hat{q}_{t}$ and $h_{t}=\frac{-c(m-1) z(m-1)}{c(m-2) z(m-2)} \hat{r}_{t}<0$ making the right-hand side a negative Nevanlinna function, i.e. we obtain (3.38). 
If $z(n)$ does obey (3.37) for $\lambda=0$, then $\frac{z(m-1)}{z(m)}=R_{s, m}^{0}(0)$. Thus, one of $\hat{q}_{t}{ }^{\prime} \mathrm{s}, t=1, \ldots, p$, is equal to 0 . As in Theorems 3.2 and 3.3, it can be shown that the singularity at $\lambda=0$ is removable giving that the number of non-removable $\hat{q}_{t}$ 's is equal to the number of $\rho_{k}$ 's.

Therefore, in (3.47) $p=s-1$. So after relabeling, if necessary, (3.47) becomes

$$
\hat{f}(\lambda)-\sum_{t=1}^{s-1} \frac{\hat{r}_{t}}{\lambda-\hat{q}_{t}}, \quad \hat{r}_{t}>0
$$

As before, for $\lambda \rightarrow \pm \infty$, we have that $\frac{1}{R_{s, m}^{0}(\lambda)} \rightarrow \omega \lambda+\xi$, thus again $\hat{f}(\lambda)=-\frac{1}{\omega}$. As a result,

$$
\frac{\nu(m-2)}{v(m-1)}:=\phi-\sum_{t=1}^{s-1} \frac{\tilde{h}_{t}}{\lambda-\tilde{g}_{t}}
$$

where $\phi$ is as defined above, $\tilde{g}_{t}=\hat{q}_{t}$ and $\tilde{h}_{t}=\frac{-c(m-1) z(m-1)}{c(m-2) z(m-2)} \hat{r}_{t}<0$, i.e. a negative Nevanlinna function of the form (3.39).

\subsection{Boundary condition at the initial end point}

For transformation (2.1), the theorem below could be considered analogous to Theorems 3.2 and 3.3 given in [2] for the reverse transformation.

Theorem 3.5 Consider the boundary condition

$$
\begin{aligned}
y(-1) & =\left[g \lambda+h-\sum_{k=1}^{p} \frac{u_{k}}{\lambda-e_{k}}\right] y(0), \quad g \geq 0, u_{k}>0 \\
& :=R_{p,-1}^{\diamond}(\lambda) y(0) \quad \text { for } \diamond= \begin{cases}+ & \text { if } g>0, \\
0 & \text { if } g=0 .\end{cases}
\end{aligned}
$$

Under mapping (2.1), $y(n)$ obeying (3.50) transforms to $v(n)$ obeying boundary conditions of the following form:

(A) If $z(n)$ does not obey (3.50) for $\lambda=0$, then $v(n)$ obeys a boundary condition of the form

(i)

$$
v(-1)=\left[-\sum_{t=1}^{p+1} \frac{\gamma_{t}}{\lambda-v_{t}}\right] \nu(0):=T_{p+1,-1}^{0}(\lambda) v(0), \quad g=0, h<\frac{z(-1)}{z(0)}
$$

(ii)

$$
v(-1)=\left[\tilde{\beta}-\sum_{t=1}^{p+1} \frac{\tilde{\gamma}_{t}}{\lambda-\tilde{v}_{t}}\right] \nu(0):=\tilde{T}_{p+1,-1}^{0}(\lambda) v(0), \quad g>0 .
$$

(B) If $z(n)$ does obey (3.50) for $\lambda=0$, then $v(n)$ obeys a boundary condition of the form

(i)

$$
v(-1)=\left[-\sum_{t=1}^{p} \frac{\hat{\gamma}_{t}}{\lambda-\hat{v}_{t}}\right] \nu(0):=\hat{T}_{p,-1}^{0}(\lambda) v(0), \quad g=0, h<\frac{z(-1)}{z(0)}
$$


(ii)

$$
v(-1)=\left[\bar{\beta}-\sum_{t=1}^{p} \frac{\bar{\gamma}_{t}}{\lambda-\bar{v}_{t}}\right] \nu(0):=\bar{T}_{p,-1}^{0}(\lambda) v(0), \quad g>0,
$$

where $\gamma_{t}, \tilde{\gamma}_{t}, \hat{\gamma}_{t}, \bar{\gamma}_{t}>0$, i.e. $T_{p+1,-1}^{0}(\lambda), \tilde{T}_{p+1,-1}^{0}(\lambda), \hat{T}_{p,-1}^{0}(\lambda), \bar{T}_{p,-1}^{0}(\lambda)$ are positive Nevanlinna functions.

Proof Using (2.1) with $n=-1$ and 0 together with (3.50) gives

$$
v(-1)=y(0)\left[1-\frac{z(0)}{z(-1)} R_{p,-1}^{\diamond}(\lambda)\right]
$$

and

$$
v(0)=y(1)-y(0) \frac{z(1)}{z(0)}
$$

Solving for $y(1)$ from equation (1.1) with $n=0$ and then substituting into (3.56) gives

$$
v(0)=y(0)\left\{\frac{1}{c(0)}\left[b(0)-\lambda c(0)-c(-1) R_{p,-1}^{\diamond}(\lambda)\right]-\frac{z(1)}{z(0)}\right\} .
$$

Hence from (3.55) and (3.57) it follows that

$$
\frac{v(-1)}{v(0)}=\frac{1-\frac{z(0)}{z(-1)} R_{p,-1}^{\diamond}(\lambda)}{\frac{b(0)}{c(0)}-\lambda-\frac{c(-1)}{c(0)} R_{p,-1}^{\diamond}(\lambda)-\frac{z(1)}{z(0)}}
$$

and after simplification this becomes

$$
\frac{v(-1)}{v(0)}=\frac{z(0)}{z(-1)}\left[\frac{1}{\frac{c(-1)}{c(0)}-\frac{\lambda}{\frac{z(-1)}{z(0)}-R_{p,-1}^{\diamond}(\lambda)}}\right] .
$$

Now

$$
\frac{\lambda}{\frac{z(-1)}{z(0)}-R_{p,-1}^{\diamond}(\lambda)}
$$

has the expansion

$$
\bar{f}(\lambda)-\sum_{t=1}^{k} \frac{\bar{r}_{t}}{\lambda-\bar{q}_{t}}
$$

where $\bar{q}_{t}$ corresponds to the singularities of (3.58), that is, where $R_{p,-1}^{\diamond}(\lambda)=\frac{z(-1)}{z(0)}$.

If $g=0$ in (3.50), then the graph has the form shown in Figure 1 where $y(\lambda)=R_{p,-1}^{0}(\lambda)$, $K=\frac{z(-1)}{z(0)}, A=0, B=h$ such that $0<B<K, x_{i}=e_{i}$ and $\tau_{i}=\bar{q}_{i}$ for $i=1,2,3$. Note that since $A=0, \tau_{4}$ does not exist. 
Clearly, the gradient of $R_{p,-1}^{0}(\lambda)$ at $\bar{q}_{t}$ is positive for all $t$, that is,

$$
\left.\frac{\partial}{\partial \lambda} R_{p,-1}^{0}(\lambda)\right|_{\bar{q}_{t}}>0, \quad t=1, \ldots, k
$$

If $z(n)$ does not obey (3.50) when $g=0$, then the zeros of $\frac{\lambda}{\frac{z(-1)}{z(0)}-R_{p,-1}^{0}(\lambda)}$ are the poles of $R_{p,-1}^{0}(\lambda)$, that is, $e_{j}$ 's together with $\lambda=0$. Since there is the same number of $\bar{q}_{t}$ 's as there are $e_{j}$ 's, it follows that $k=p$ in (3.60).

We next examine the form of $\bar{f}(\lambda)$ in (3.60). Now

$$
\frac{\lambda}{\frac{z(-1)}{z(0)}-R_{p,-1}^{0}(\lambda)} \rightarrow \frac{\lambda}{\frac{z(-1)}{z(0)}-h}
$$

Therefore, $\bar{f}(\lambda)=\frac{\lambda}{\frac{z(-1)}{z(0)}-h}$. Hence, using (3.58), we obtain

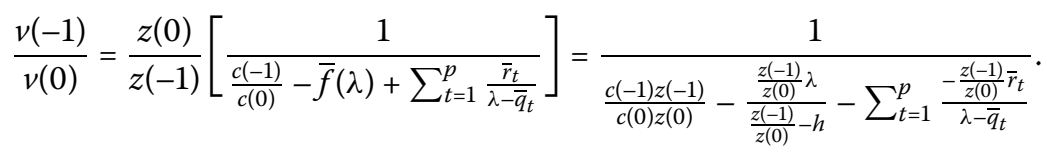

It should be noted that $\frac{\frac{z(-1)}{z(0)}}{\frac{z(-1)}{z(0)}-h}>0$ when $\frac{z(-1)}{z(0)}>h$ and $-\frac{z(-1)}{z(0)} \bar{r}_{t}<0$. Thus

$$
\frac{\nu(-1)}{v(0)}=\frac{1}{N_{p,-1}^{-}(\lambda)}
$$

where $N_{p,-1}^{-}(\lambda)$ is a negative Nevanlinna function, and using Nevanlinna property $(C)$ the right-hand side can be re-written to give

$$
\frac{\nu(-1)}{v(0)}=-\sum_{t=1}^{p+1} \frac{\gamma_{t}}{\lambda-v_{t}}, \quad \gamma_{t}>0
$$

which is of the form required by (3.51).

If $g=0$ and $z(n)$ obeys (3.50) for $\lambda=0$, then $\frac{z(-1)}{z(0)}=R_{p,-1}^{0}(\lambda)$. Thus, one of $\bar{q}_{t}$ 's, $t=1, \ldots, k$, is zero, and hence, by (3.50) with $g=0$,

$$
\frac{\lambda}{\frac{z(-1)}{z(0)}-R_{p,-1}^{0}(\lambda)}=\frac{1}{\sum_{k=1}^{p} \frac{u_{k} / e_{k}}{\lambda-e_{k}}}
$$

so that once again the discontinuity at zero has been removed. This means that the number of non-removable discontinuities, i.e. $\bar{q}_{t}$ 's, is one less than the number of $e_{k}$ 's, that is, in (3.60) put $k=p-1$.

Exactly the same procedure, as used for the case of $z(n)$ not obeying (3.50) for $g=0$, may be used with $p$ replaced by $p-1$ to obtain (3.53), that is,

$$
\frac{v(-1)}{v(0)}=-\sum_{t=1}^{p} \frac{\hat{\gamma}_{t}}{\lambda-\hat{v}_{t}}, \quad \hat{\gamma}_{t}>0
$$


If, in equation (3.50), $g>0$, then the graph of $R_{p,-1}^{+}(\lambda)$ has the form shown in Figure 1 where $y(\lambda)=R_{p,-1}^{+}(\lambda), K=\frac{z(-1)}{z(0)}, A=g, B=h, x_{j}=e_{j}$ for $j=1,2,3$ and $\tau_{i}=\bar{q}_{i}$ for $i=1,2,3,4$. It is evident that the gradient of $R_{p,-1}^{+}(\lambda)$ is positive at $\bar{q}_{t}$ 's.

If $z(n)$ does not obey (3.50) when $g>0$, then the zeros of $\frac{\lambda}{\frac{z(-1)}{z(0)}-R_{p,-1}^{+}(\lambda)}$ are the poles of $R_{p,-1}^{+}(\lambda)$, that is, $e_{k}$ 's together with $\lambda=0$. We see that the number of $\bar{q}_{t}$ 's is one more than the number of $e_{k}$ 's, so in (3.60) we have $k=p+1$. In terms of the behavior as $\lambda \rightarrow \pm \infty$, we obtain

$$
\frac{\lambda}{\frac{z(-1)}{z(0)}-R_{p,-1}^{+}(\lambda)} \rightarrow \frac{\lambda}{\frac{z(-1)}{z(0)}-(g \lambda+h)} \rightarrow-\frac{1}{g} .
$$

Thus from (3.58), (3.59) and (3.60) it follows that

$$
\frac{v(-1)}{v(0)}=\frac{1}{\frac{c(-1) z(-1)}{c(0) z(0)}+\frac{1}{g} \frac{z(-1)}{z(0)}-\sum_{t=1}^{p+1} \frac{\frac{-z(-1)}{z(0)} \bar{r}_{t}}{\lambda-\bar{q}_{t}}} .
$$

Now

$$
\frac{z(-1)}{z(0)}\left[\frac{c(-1)}{c(0)}+\frac{1}{g}\right]=0 \Rightarrow \frac{c(-1)}{c(0)}=-\frac{1}{g}
$$

which is not possible since $g>0$ and $\frac{c(-1)}{c(0)}>0$. Therefore,

$$
\frac{v(-1)}{v(0)}=\frac{1}{\Gamma-\sum_{t=1}^{p+1} \frac{p_{t}}{\lambda-\bar{q}_{t}}},
$$

where $\Gamma=\frac{z(-1)}{z(0)}\left[\frac{c(-1)}{c(0)}+\frac{1}{g}\right] \neq 0$ and $p_{t}=\frac{-z(-1)}{z(0)} \bar{r}_{t}<0$. Hence, by Nevanlinna property (B), we obtain (3.52), that is,

$$
\frac{v(-1)}{v(0)}=\tilde{\beta}-\sum_{t=1}^{p+1} \frac{\tilde{\gamma}_{t}}{\lambda-\tilde{v}_{t}}, \quad \tilde{\gamma}_{t}>0
$$

where $\tilde{\beta}=\frac{1}{\Gamma} \neq 0$.

As usual, if $z(n)$ obeys boundary condition (3.50) for $g>0$, then one of $\bar{q}_{t}$ 's is zero, and this discontinuity is removable so that the number of $\bar{q}_{t}$ 's is equal to the number of $e_{k}$ 's, that is, in (3.60) the number of terms in the sum is $p$. Using the method outlined above for the case of $z(n)$ not obeying (3.50) for $g>0$, with $p$ replaced by $p-1$, gives equation (3.54) which is

$$
\frac{v(-1)}{v(0)}=\bar{\beta}-\sum_{t=1}^{p} \frac{\bar{\gamma}_{t}}{\lambda-\bar{v}_{t}}, \quad \bar{\gamma}_{t}>0
$$

\section{Conclusion}

In conclusion, as a direct consequence of Theorems 3.2, 3.3, 3.4 and 3.5, we have Tables 1 and 2 in which we compare the original boundary value problem with the transformed boundary value problem for various sets of boundary conditions, and the corresponding number of eigenvalues is given. 
Table 1 Boundary condition (3.50) with $g=0$

\begin{tabular}{|c|c|c|}
\hline & Original BVP: (1.1) with bc's ... & Trans. BVP: (2.2) with bc's ... \\
\hline 1 & $\begin{array}{l}\text { (3.20) and (3.50) with } g=0 \\
z \text { does not obey (3.20) or (3.50) } \\
s+p+m \text { eigenvalues }\end{array}$ & $\begin{array}{l}\text { (3.21) and (3.51) } \\
s+1+p+1+m-1 \text { eigenvalues } \\
\text { i.e. one extra eigenvalue } 0\end{array}$ \\
\hline 2 & $\begin{array}{l}\text { (3.20) and (3.50) with } g=0 \\
z \text { obeys (3.20) but not }(3.50) \\
s+p+m \text { eigenvalues }\end{array}$ & $\begin{array}{l}\text { (3.22) and (3.51) } \\
s+p+1+m-1 \text { eigenvalues } \\
\text { i.e. same eigenvalues }\end{array}$ \\
\hline 3 & $\begin{array}{l}\text { (3.20) and ( } 3.50) \text { with } g=0 \\
z \text { obeys ( } 3.50) \text { but not }(3.20) \\
s+p+m \text { eigenvalues }\end{array}$ & $\begin{array}{l}\text { (3.21) and ( } 3.53) \\
s+1+p+m-1 \text { eigenvalues } \\
\text { i.e. same eigenvalues }\end{array}$ \\
\hline 4 & $\begin{array}{l}\text { (3.20) and ( } 3.50) \text { with } g=0 \\
z \text { obeys both ( } 3.20) \text { and ( } 3.50) \\
s+p+m \text { eigenvalues }\end{array}$ & $\begin{array}{l}\text { (3.22) and (3.53) } \\
s+p+m-1 \text { eigenvalues } \\
\text { i.e. one less eigenvalue } 0\end{array}$ \\
\hline 5 & $\begin{array}{l}\text { (3.28) and (3.50) with } g=0 \\
z \text { does not obey (3.28) or (3.50), } a=\frac{-c(m)}{c(m-1)} \\
s+p+m+1 \text { eigenvalues }\end{array}$ & $\begin{array}{l}\text { (3.29) and (3.51) } \\
s+p+1+m+1 \text { eigenvalues } \\
\text { i.e. one extra eigenvalue } 0\end{array}$ \\
\hline 6 & $\begin{array}{l}\text { (3.28) and (3.50) with } g=0 \\
z \text { obeys (3.28) but not (3.50), } a=\frac{-c(m)}{c(m-1)} \\
s+p+m+1 \text { eigenvalues }\end{array}$ & $\begin{array}{l}\text { (3.31) and (3.51) } \\
s-1+p+1+m+1 \text { eigenvalues } \\
\text { i.e. same eigenvalues }\end{array}$ \\
\hline 7 & $\begin{array}{l}\text { (3.28) and (3.50) with } g=0 \\
z \text { obeys (3.50) but not (3.28), } a=\frac{-c(m)}{c(m-1)} \\
s+p+m+1 \text { eigenvalues }\end{array}$ & $\begin{array}{l}\text { (3.29) and (3.53) } \\
s+p+m+1 \text { eigenvalues } \\
\text { i.e. same eigenvalues }\end{array}$ \\
\hline 8 & $\begin{array}{l}\text { (3.28) and (3.50) with } g=0 \\
z \text { obeys both (3.28) and (3.50), } a=\frac{-c(m)}{c(m-1)} \\
s+p+m+1 \text { eigenvalues }\end{array}$ & $\begin{array}{l}\text { (3.31) and (3.53) } \\
s-1+p+m+1 \text { eigenvalues } \\
\text { i.e. one less eigenvalue } 0\end{array}$ \\
\hline 9 & $\begin{array}{l}\text { (3.28) and (3.50) with } g=0 \\
z \text { does not obey (3.28) or (3.50), } a \neq \frac{-c(m)}{c(m-1)} \\
s+p+m+1 \text { eigenvalues }\end{array}$ & $\begin{array}{l}\text { (3.30) and (3.51) } \\
s+1+p+1+m \text { eigenvalues } \\
\text { i.e. one extra eigenvalue } 0\end{array}$ \\
\hline 10 & $\begin{array}{l}\text { (3.28) and (3.50) with } g=0 \\
z \text { obeys (3.28) but not (3.50), } a \neq \frac{-c(m)}{c(m-1)} \\
s+p+m+1 \text { eigenvalues }\end{array}$ & $\begin{array}{l}\text { (3.32) and (3.51) } \\
s+p+1+m \text { eigenvalues } \\
\text { i.e. same eigenvalues }\end{array}$ \\
\hline 11 & $\begin{array}{l}\text { (3.28) and (3.50) with } g=0 \\
z \text { obeys (3.50) but not (3.28), } a \neq \frac{-c(m)}{c(m-1)} \\
s+p+m+1 \text { eigenvalues }\end{array}$ & $\begin{array}{l}\text { (3.30) and ( } 3.53) \\
s+1+p+m \text { eigenvalues } \\
\text { i.e. same eigenvalues }\end{array}$ \\
\hline 12 & $\begin{array}{l}\text { (3.28) and (3.50) with } g=0 \\
z \text { obeys both (3.28) and (3.50), } a \neq \frac{-c(m)}{c(m-1)} \\
s+p+m+1 \text { eigenvalues }\end{array}$ & $\begin{array}{l}\text { (3.32) and (3.53) } \\
s+p+m \text { eigenvalues } \\
\text { i.e. one less eigenvalue } 0\end{array}$ \\
\hline 13 & $\begin{array}{l}\text { (3.37) and (3.50) with } g=0 \\
z \text { does not obey (3.37) or ( } 3.50) \\
s+p+m-1 \text { eigenvalues }\end{array}$ & $\begin{array}{l}\text { (3.38) and (3.51) } \\
s+p+1+m-1 \text { eigenvalues } \\
\text { i.e. one extra eigenvalue } 0\end{array}$ \\
\hline 14 & $\begin{array}{l}\text { (3.37) and (3.50) with } g=0 \\
z \text { obeys (3.37) but not ( } 3.50) \\
s+p+m-1 \text { eigenvalues }\end{array}$ & $\begin{array}{l}\text { (3.39) and (3.51) } \\
s-1+p+1+m-1 \text { eigenvalues } \\
\text { i.e. same eigenvalues }\end{array}$ \\
\hline 15 & $\begin{array}{l}\text { (3.37) and (3.50) with } g=0 \\
z \text { obeys (3.50) but not ( } 3.37) \\
s+p+m-1 \text { eigenvalues }\end{array}$ & $\begin{array}{l}\text { (3.38) and (3.53) } \\
s+p+m-1 \text { eigenvalues } \\
\text { i.e. same eigenvalues }\end{array}$ \\
\hline 16 & $\begin{array}{l}\text { (3.37) and (3.50) with } g=0 \\
z \text { obeys both ( } 3.37) \text { and ( } 3.50) \\
s+p+m-1 \text { eigenvalues }\end{array}$ & $\begin{array}{l}\text { (3.39) and ( } 3.53) \\
s-1+p+m-1 \text { eigenvalues } \\
\text { i.e. one less eigenvalue } 0\end{array}$ \\
\hline
\end{tabular}


Table 2 Boundary condition (3.50) with $g>0$

\begin{tabular}{|c|c|c|}
\hline & Original BVP: (1.1) with bc's ... & Trans. BVP: (2.2) with bc's ... \\
\hline 1 & $\begin{array}{l}\text { (3.20) and (3.50) with } g>0 \\
z \text { does not obey (3.20) or ( } 3.50) \\
s+p+m \text { eigenvalues }\end{array}$ & $\begin{array}{l}\text { (3.21) and (3.52) } \\
s+1+p+1+m-1 \text { eigenvalues } \\
\text { i.e. one extra eigenvalue } 0\end{array}$ \\
\hline 2 & $\begin{array}{l}\text { (3.20) and (3.50) with } g>0 \\
z \text { obeys (3.20) but not }(3.50) \\
s+p+m \text { eigenvalues }\end{array}$ & $\begin{array}{l}(3.22) \text { and }(3.52) \\
s+p+1+m-1 \text { eigenvalues } \\
\text { i.e. same eigenvalues }\end{array}$ \\
\hline 3 & $\begin{array}{l}\text { (3.20) and (3.50) with } g>0 \\
z \text { obeys (3.50) but not }(3.20) \\
s+p+m \text { eigenvalues }\end{array}$ & $\begin{array}{l}\text { (3.21) and (3.54) } \\
s+1+p+m-1 \text { eigenvalues } \\
\text { i.e. same eigenvalues }\end{array}$ \\
\hline 4 & $\begin{array}{l}\text { (3.20) and (3.50) with } g>0 \\
z \text { obeys both ( } 3.20) \text { and ( } 3.50) \\
s+p+m \text { eigenvalues }\end{array}$ & $\begin{array}{l}\text { (3.22) and (3.54) } \\
s+p+m-1 \text { eigenvalues } \\
\text { i.e. one less eigenvalue } 0\end{array}$ \\
\hline 5 & $\begin{array}{l}\text { (3.28) and (3.50) with } g>0 \\
z \text { does not obey (3.28) or (3.50), } a=\frac{-c(m)}{c(m-1)} \\
s+p+m+1 \text { eigenvalues }\end{array}$ & $\begin{array}{l}\text { (3.29) and (3.52) } \\
s+p+1+m+1 \text { eigenvalues } \\
\text { i.e. one extra eigenvalue } 0\end{array}$ \\
\hline 6 & $\begin{array}{l}\text { (3.28) and (3.50) with } g>0 \\
z \text { obeys (3.28) but not (3.50), } a=\frac{-c(m)}{c(m-1)} \\
s+p+m+1 \text { eigenvalues }\end{array}$ & $\begin{array}{l}\text { (3.31) and (3.52) } \\
s-1+p+1+m+1 \text { eigenvalues } \\
\text { i.e. same eigenvalues }\end{array}$ \\
\hline 7 & $\begin{array}{l}\text { (3.28) and (3.50) with } g>0 \\
z \text { obeys (3.50) but not (3.28), } a=\frac{-c(m)}{c(m-1)} \\
s+p+m+1 \text { eigenvalues }\end{array}$ & $\begin{array}{l}\text { (3.29) and (3.54) } \\
s+p+m+1 \text { eigenvalues } \\
\text { i.e. same eigenvalues }\end{array}$ \\
\hline 8 & $\begin{array}{l}\text { (3.28) and (3.50) with } g>0 \\
z \text { obeys both (3.28) and (3.50), } a=\frac{-c(m)}{c(m-1)} \\
s+p+m+1 \text { eigenvalues }\end{array}$ & $\begin{array}{l}(3.31) \text { and (3.54) } \\
s-1+p+m+1 \text { eigenvalues } \\
\text { i.e. one less eigenvalue } 0\end{array}$ \\
\hline 9 & $\begin{array}{l}\text { (3.28) and (3.50) with } g>0 \\
z \text { does not obey (3.28) or (3.50), } a \neq \frac{-c(m)}{c(m-1)} \\
s+p+m+1 \text { eigenvalues }\end{array}$ & $\begin{array}{l}\text { (3.30) and (3.52) } \\
s+1+p+1+m \text { eigenvalues } \\
\text { i.e. one extra eigenvalue } 0\end{array}$ \\
\hline 10 & $\begin{array}{l}\text { (3.28) and (3.50) with } g>0 \\
z \text { obeys (3.28) but not (3.50), } a \neq \frac{-c(m)}{c(m-1)} \\
s+p+m+1 \text { eigenvalues }\end{array}$ & $\begin{array}{l}\text { (3.32) and (3.52) } \\
s+p+1+m \text { eigenvalues } \\
\text { i.e. same eigenvalues }\end{array}$ \\
\hline 11 & $\begin{array}{l}\text { (3.28) and (3.50) with } g>0 \\
z \text { obeys (3.50) but not (3.28), } a \neq \frac{-c(m)}{c(m-1)} \\
s+p+m+1 \text { eigenvalues }\end{array}$ & $\begin{array}{l}\text { (3.30) and ( } 3.54) \\
s+1+p+m \text { eigenvalues } \\
\text { i.e. same eigenvalues }\end{array}$ \\
\hline 12 & $\begin{array}{l}\text { (3.28) and (3.50) with } g>0 \\
z \text { obeys both (3.28) and (3.50), } a \neq \frac{-c(m)}{c(m-1)} \\
s+p+m+1 \text { eigenvalues }\end{array}$ & $\begin{array}{l}\text { (3.32) and (3.54) } \\
s+p+m \text { eigenvalues } \\
\text { i.e. one less eigenvalue } 0\end{array}$ \\
\hline 13 & $\begin{array}{l}\text { (3.37) and (3.50) with } g>0 \\
z \text { does not obey (3.37) or (3.50) } \\
s+p+m-1 \text { eigenvalues }\end{array}$ & $\begin{array}{l}\text { (3.38) and (3.52) } \\
s+p+1+m-1 \text { eigenvalues } \\
\text { i.e. one extra eigenvalue } 0\end{array}$ \\
\hline 14 & $\begin{array}{l}\text { (3.37) and (3.50) with } g>0 \\
z \text { obeys (3.37) but not }(3.50) \\
s+p+m-1 \text { eigenvalues }\end{array}$ & $\begin{array}{l}\text { (3.39) and (3.52) } \\
s-1+p+1+m-1 \text { eigenvalues } \\
\text { i.e. same eigenvalues }\end{array}$ \\
\hline 15 & $\begin{array}{l}\text { (3.37) and (3.50) with } g>0 \\
z \text { obeys (3.50) but not ( } 3.37) \\
s+p+m-1 \text { eigenvalues }\end{array}$ & $\begin{array}{l}\text { (3.38) and (3.54) } \\
s+p+m-1 \text { eigenvalues } \\
\text { i.e. same eigenvalues }\end{array}$ \\
\hline 16 & $\begin{array}{l}\text { (3.37) and (3.50) with } g>0 \\
z \text { obeys both ( } 3.37) \text { and ( } 3.50) \\
s+p+m-1 \text { eigenvalues }\end{array}$ & $\begin{array}{l}\text { (3.39) and (3.54) } \\
s-1+p+m-2 \text { eigenvalues } \\
\text { i.e. one less eigenvalue } 0\end{array}$ \\
\hline
\end{tabular}

Remark To summarize, we have the following:

(a) If $z(n)$ obeys the boundary conditions at both ends, then the transformed boundary value problem loses the eigenvalue 0 but retains the remaining eigenvalues from the original boundary value problem;

(b) If $z(n)$ obeys the boundary condition at one end only, then the transformed 
boundary value problem will have exactly the same eigenvalues as the original boundary value problem;

(c) If $z(n)$ does not obey any of the boundary conditions, then the transformed boundary value problem gains the eigenvalue 0 , with corresponding eigenfunction $\frac{1}{c(n) z(n)}$, in addition to the eigenvalues of the original boundary value problem.

The above remark is consistent with the results obtained in $[2,3]$ and may be proved in the same way as [2, Corollary 4.4], see also [3].

We conclude with the following observation. Since the reverse transformation used in [2] was at $n$ and $n-1$, whereas in this paper the forward transformation is at $n$ and $n+1$, the roles of the endpoints are in a sense 'reversed' which is well illustrated by the results obtained above.

Competing interests

The authors declare that they have no competing interests.

Authors' contributions

SC and ADL contributed equally to all aspects of the work. Both authors read and approved the final manuscript.

\section{Acknowledgements}

The first author is supported by NRF grant no. IFR2011040100017.

Received: 7 June 2013 Accepted: 11 October 2013 Published: 08 Nov 2013

\section{References}

1. Currie, S, Love, A: Transformations of difference equations I. Adv. Differ. Equ. 2010, Article ID 947058 (2010)

2. Currie, S, Love, A: Hierarchies of difference boundary value problems. Bound. Value Probl. 2011, Article ID 743135 (2011)

3. Currie, S, Love, A: Erratum to: Hierarchies of difference boundary value problems. Bound. Value Probl. 2012, Article ID $66(2012)$

4. Currie, S, Love, A: Hierarchies of difference boundary value problems continued. J. Differ. Equ. Appl. (2013). doi:10.1080/10236198.2013.778841

5. Elaydi, S: An Introduction to Difference Equations. Springer, Berlin (2005)

6. Harmsen, B, Li, A: Discrete Sturm-Liouville problems with parameter in the boundary conditions. J. Differ. Equ. Appl. 8(11), 869-891 (2002)

7. Harmsen, B, Li, A: Discrete Sturm-Liouville problems with nonlinear parameter in the boundary conditions. J. Differ. Equ. Appl. 13(7), 639-653 (2007)

8. Maeda, S: The similarity method for difference equations. IMA J. Appl. Math. 38, 129-134 (1987)

9. Quispel, GRW, Sahadevan, R: Lie symmetries and the integration of difference equations. Phys. Lett. A 184, 64-70 (1993)

10. Hydon, PE: Symmetries and first integrals of ordinary difference equations. Proc. R. Soc. Lond. A 454, 2835-2855 (2000)

11. Levi, D, Winternitz, P: Continuous symmetries of difference equations. J. Math. Phys. 39, R1-R63 (2005)

10.1186/1687-1847-2013-311

Cite this article as: Currie and Love: Difference boundary value problem hierarchies and the forward Crum transformation. Advances in Difference Equations 2013, 2013:311 